\title{
On the representation of intentions: Do personally relevant consequences determine activation?
}

\author{
Janette C. Schult • Melanie C. Steffens
}

Published online: 18 May 2011

(C) Psychonomic Society, Inc. 2011

\begin{abstract}
The intention-superiority effect describes shorter latencies for reactions to stimuli intended for future enactment, relative to stimuli associated with no enactment or canceled enactment. Previous attempts to demonstrate an intention-superiority effect for other types of tasks-for instance, observing the experimenter executing actionshave not yielded an intention-superiority effect. A reason for this could be that the typical enactment task was associated with a higher degree of personal relevance than were other laboratory-based tasks and that task importance or its consequences heighten the accessibility of intentionrelevant materials. In two experiments, we demonstrate an intention-superiority effect for different types of tasks (e.g., monitoring a video clip) when task realization has personally relevant consequences in terms of a performance evaluation. In contrast, we found no intention-superiority effect when future enactment had no personally relevant consequences for participants. These findings imply that the intention-superiority effect is not restricted to actions but occurs generally for relevant plans.
\end{abstract}

Keywords Memory Intention · Lexical-decision ·

Relevance

Prospective memory refers to the ability to realize an intended task at an appropriate moment in the future. A key feature of prospective memory tasks, such as passing on a message to a colleague when seeing her, buying milk on one's way home, or attending the yearly routine check at the dentist, is that task

J. C. Schult $(\bowtie) \cdot$ M. C. Steffens

Institut für Psychologie, Friedrich-Schiller-Universität Jena,

Am Steiger 3, Haus 1,

07743 Jena, Germany

e-mail: janette.schult@uni-jena.de execution is postponed for hours, days, or even weeks or months. During postponement, a representation of the intended task must be retained until an appropriate opportunity to fulfill the task occurs (e.g., Ellis, 1996). Previous research has shown that stimuli describing the actions intended for future enactment remain activated during postponement. The aim of the present research was testing whether this finding is more general, extending to all intention-relevant information, be it related to the execution of actions or not.

In 1993, Goschke and Kuhl introduced a paradigm for examining the accessibility of intention-relevant materials during postponement. Participants learned two short lists of actions (e.g., "setting a dinner table" and "clearing a messy desk") for a later recognition test. After the study phase, they were informed that they should carry out the actions of one list (prospective list), but not of the other list (neutral list), after a recognition test for both lists. In the recognition test, response latencies for verbs and nouns on the prospective list were shorter than latencies for the stimuli on the neutral list. The authors labeled this reaction-time advantage the intention-superiority effect. The intentionsuperiority effect has been replicated with a more direct measure of accessibility, a lexical-decision task (LDT; Marsh, Hicks, \& Bink, 1998; Marsh, Hicks,\& Bryan, 1999), and in a more naturalistic setting (Dockree \& Ellis, 2001).

Different mechanisms underlying the intention-superiority effect have been discussed. For example, Goschke and Kuhl (1993) suggested that intentions are represented as subthreshold nodes in long-term memory that decay more slowly than more neutral contents. According to that view, a heightened accessibility of intention-related concepts is an intrinsic property of the representation of the intention. Alternatively, Freeman and Ellis (2003) proposed that motor or sensorimotor information associated with the future execution of actions is stored with the representation of the 
intention. Thus, the intention-superiority effect would be due to a rich multimodal representation of intentions, as compared with verbal representations (for similar reasoning, see, e.g., Engelkamp, 1997; Koriat, Ben-Zur, \& Nussbaum, 1990). Finally, intentions are related to motivational states, and thus the strength of the intention may determine the accessibility of intention-related concepts (e.g., Förster, Liberman, \& Friedman, 2007; Förster, Liberman, \& Higgins, 2005). Thus, the intention-superiority effect could reflect the participant's degree of commitment to realizing the intention. The role of motivation-related processes remains an open question in studies on the intention-superiority effect in a postponed-intention paradigm.

The existing studies suggest that the intention-superiority effect is restricted to one type of task: the future enactment of the learned actions. Stimuli on the prospective list were responded to more quickly than were stimuli on the neutral list if participants intended to carry out the actions on the prospective list after the assessment of response latencies (Dockree \& Ellis, 2001; Goschke \& Kuhl, 1993; Marsh et al., 1998; Marsh et al., 1999). If participants, however, intended to observe the experimenter executing the actions (Goschke \& Kuhl, 1993; Marsh et al., 1998), or if they were asked to verbally recall the actions (Freeman \& Ellis, 2003), response latencies were comparable for the stimuli on the prospective and the neutral lists. In order to explain this pattern of findings, we suggest that the types of laboratory-based tasks differed with regard to the degree of commitment to realizing the intention. In particular, we assume that the future enactment task was associated with a higher degree of personal relevance for the participants, as compared with an observation task or verbal recall. Thus, the intentionsuperiority effect for future enactment might reflect a higher motivation to fulfill the denoted task, rather than the multimodal representation of the intention that Freeman and Ellis (2003; see previous paragraph) postulated. There are hints for this in the previous studies.

First, in studies comparing an enactment and an observation task (Goschke \& Kuhl, 1993; Marsh et al., 1998), the incentive to fulfill the enactment task properly was higher than that to fulfill the observation task. For the enactment task, task requirements were clearly defined (enact a list of specified actions), and task realization resulted in an observable outcome that could be evaluated by the experimenter (e.g., how many or in which order actions were performed). In addition, failing to perform a short, wellknown script such as "setting a dinner table"in the presence of the experimenter might be perceived as embarrassing. Although an evaluation of participants' performance was not explicitly announced, participants were replaced who did not perform all actions (Goschke \& Kuhl, 1993) or who enacted the list in the wrong order (Marsh et al., 1998). In the respective observation condition, participants were instructed to watch the experimenter and register mistakes (Goschke \& Kuhl, 1993) or to verify whether the experimenter had performed the correct list of actions (Marsh et al., 1998). Whether they did monitor the experimenter attentively was not reported. Thus, participants could faithfully fulfill the instructions, but in contrast to the enactment task, they did not have to, and no participant was excluded due to task failure. Consequences such as an evaluation of one's monitoring performance were also rather unlikely, since task requirements were defined only vaguely. In short, the public outcome, as well as an anticipated evaluation of one's enactment performance, may have strengthened the motivation to fulfill the enactment task properly, as compared with the observation task.

A similar reasoning applies to potential differences in motivation in Freeman and Ellis's (2003) research. Participants were asked either to pantomimically enact a list of verbs or to recall them orally. Thus, for both types of tasks, task requirements were clear, and the quality of task realization was evaluated in terms of recall performance. Yet the idea of having to recall verbs such as "to eat" or "to grasp" by bodily or facial movements in the presence of the experimenter could be perceived as more unpleasant or embarrassing than verbally recalling them (Saltz, 1988). Again, this may result in an increased effort to encode or store stimuli related to future enactment, which could imply that these stimuli are more easily accessible during postponement than are those intended for verbal recall.

To sum up, prior studies have demonstrated shorter latencies for intention-related materials, relative to more neutral materials, during postponement if the materials were to be enacted (usually in the presence of an experimenter). We propose that the anticipated evaluation of one's enactment has strengthened the motivation to fulfill the enactment task properly, as compared with other tasks. Assuming that the strength of motivation increases the accessibility of intentionrelated concepts, one would expect an intention-superiority effect for more relevant tasks (such as the previously used enactment task), and the intention-superiority effect should be less pronounced for less relevant tasks (such as the previously used observation task).

\section{Experiment 1}

The primary aim of Experiment 1 was to test whether an intention-superiority effect could be demonstrated with types of laboratory tasks other than future enactment if the intended task was personally relevant for participants. The general procedure was similar to that of Dockree and Ellis (2001). Participants studied two lists of actions for future tasks. Later, one list was canceled, and the other list remained relevant for the intended task. Prior to realization 
of the intended task, response latencies for the stimuli on the prospective list and the canceled list were assessed.

The intended tasks were rather simple. Participants were asked to monitor someone else's recall and to give a school grade for recall performance. We used two types of monitoring tasks that differed only concerning the mode of presentation of the to-be-monitored materials: Half of the participants expected to monitor a videotaped performance of the prospective list of actions (comparable to an observation task in prior studies), and the other half expected to monitor a written report of the prospective list. Both tasks required no overt enactment of the studied actions, but monitoring the videotaped performance involved observing actions, whereas monitoring a written report appeared maximally unrelated to motor enactment.

To make sure that task performance (the grading decision) was personally relevant, participants were informed that they had to justify their grading decision in the presence of other participants. A public task realization should strengthen the commitment to fulfill the task (e.g., Meacham, 1988). If strength of motivation contributes to a heightened accessibility of intention-relevant materials, as we assume, we should observe faster responses to stimuli on the intention-relevant prospective list than to stimuli linked with the canceled list. There should be an intention-superiority effect for both types of monitoring tasks. In line with previous research, we also expected that the stimuli on both lists would be more easily accessible than new stimuli, because they had been encountered previously during the study phase (e.g., Dockree \& Ellis, 2001; Marsh et al., 1998; Marsh et al., 1999).

\section{Method}

Participants Thirty-three participants were recruited on campus (age: $19-37$ years, $M=24, S D=4 ; 63 \%$ women). They received financial compensation for participation $(3 €)$. One participant failed to indicate the correct prospective list and was replaced. Sixteen participants were randomly allocated to each task condition.

Materials Two lists describing simple actions (mixing a cocktail, preparing chocolate candy) served as study materials. Each study list consisted of a title (e.g., mixing a cocktail) and four typical actions (measure the amount of alcohol, pour everything into a cocktail shaker, shake well with both hands, strain into a glass). The LDT included words from the two study lists, distractor words, and nonwords. From each study list, four nouns and four verbs were chosen. The distractor words consisted of eight words related to another theme (cooking a sushi dish) and eight unrelated words (four nouns and four verbs). Distractor words did not differ in word length or word frequency from studied ones. Nonwords consisted of 32 pronounceable letter strings that were matched on word length with words. Half of the nonwords had typical German verb endings (e.g., ieren). A further 12 stimuli (six words, six nonwords) were used as buffer items that preceded the critical lexicaldecision trials. The critical trials were presented in a random order, with the restriction that half of each type of item was presented in the first or second half of the LDT.

As to-be-monitored materials, a video clip, as well as a written report of each study list, was constructed. Materials were incomplete, and the order of actions was incoherent, allowing flexibility in grading decisions. The errors contained in the video clips and the written reports of each list were identical. All materials were prepared for presentation via a video projector. A simple yes-no checklist (Was the correct list remembered? Were all actions remembered? Were the actions remembered in a correct order?) was prepared to guide grading decisions.

Procedure Participants were tested in groups of 2-4. They were told that we were interested mainly in their ability to evaluate others' memory performance correctly. They were going to learn two lists of actions, and later they would see a written recall test for one list and a videotaped performance test for the other list. Their main job was to give school grades for recall performance in those tests and, subsequently, justify their grading decision. Thus, task realization itself (the grading decision) was private, but the quality of task performance had relevant consequences (being able to give other participants a good reason for one's decision). Instructions were given in written form and were reiterated by the experimenter. Then the LDT was introduced as a computer task and was practiced for eight trials.

Next, participants studied the two lists of actions. The lists were given on separate sheets, and the order of presentation was randomized for each participant. Study time was restricted to $1.5 \mathrm{~min}$. The experimenter informed participants about the remaining study time every $30 \mathrm{~s}$. The lists were then removed. A pretest had shown that the study time was sufficient to learn both lists. During the study phase, participants expected that both lists would be important for prospective monitoring tasks, but they did not know which list was used for what type of task until after the study phase. The encoding of a particular list for a specific task was therefore unlikely.

As a delay between list learning and retrieval, the experimenter initiated a get-to-know-each-other task that lasted about $5 \mathrm{~min}$. Then the experimenter canceled one task (e.g., monitor a written report) and one list (e.g., mixing a cocktail), allegedly due to time constraints. The other task (e.g., monitor a video clip) and the second learned list (e.g., preparing chocolate candy) remained 
relevant for the prospective task. Instructions were modeled after Dockree and Ellis (2001) - for example, in the video clip condition: "The get-to-know-each-other lasted relatively long. Because of time constraints, you are to carry out only one task: Monitoring a video clip. You no longer are to grade the written report [list A], but to grade the videotaped performance [list B]. Please attend the computer task, while I prepare everything." The assignment of prospective and canceled lists was counterbalanced across participants. We also counterbalanced the order of both half-sentences (i.e., half of the participants were told first what to do and second what not to do, and vice versa). While participants worked on the computer task (the LDT), the experimenter prepared a video projector.

Instead of presenting the to-be-monitored materials immediately after the LDT, participants were first asked to write down what they were supposed to do (monitor a video clip or a written report) as a manipulation check. Next, an unexpected free recall test for both lists was given. Participants were instructed to write down "all actions of the list you have to monitor," then "all actions of the second list you learned." Recall time was restricted to $1 \mathrm{~min}$ for each list. After recall of the second list, the video clip or the respective written report for the prospective list was presented. Participants completed the simple yes-no checklist individually and wrote down a school grade for overall memory performance. Afterwards, they compared and discussed their grading decision with other participants. Finally, they completed a short questionnaire including importance of the intended task ("A good performance at the task is . . . 1 'not at all important'-7 'very important"') and demographic data (age, sex, nationality) and were debriefed.

Design The task (monitor a video clip vs. monitor a written report) was manipulated between subjects. For all participants, performance in the respective task was relevant because a public discussion of one's grading decision was expected. The word type (prospective list, canceled list, distractor) was manipulated within subjects. The main dependent variable was response latency in the lexical-decision task.

\section{Results}

For all statistical analyses, the Type-I-error was set at $\alpha=$ .05. As an indicator of the effect size, partial $R^{2}\left(R_{\mathrm{p}}{ }^{2}\right)$ is reported for statistically significant effects (Cohen, 1977).

Preliminary analyses As a manipulation check, we inspected the importance ratings of the tasks. Participants in both groups rated a good performance at the respective monitoring tasks as equally important $(M=5.2$ for the video clip and $M=5.3$ for the written report, $t<1$ ). All free recall data were analyzed using a liberal scoring criterion: Phrases were counted as correct if original or synonymous nouns and verbs were written down. For a grading decision concerning the video clip, a verbatim recall of the studied actions would not be necessary, and we did not instruct participants to recall actions word for word. The majority (out of 16, 14 for the video clip, 11 for the written report) recalled both lists perfectly. The remaining 7 participants remembered at least three actions of each list. Thus, study time was sufficient to learn both lists equally well, and any differences in LDT latencies would reflect differences in accessibility of intention-related versus neutral materials, rather than differences in memory accuracy. If participants with imperfect recall were excluded from the subsequent analyses, the reported pattern of results did not change.

Lexical-decision task In all experiments, incorrect trials and latencies beyond $3 S D$ s of each participant's individual mean response were excluded from analyses of lexical decision data, and statistical analyses were carried out on log-transformed scores. In Experiment 1, 2.9\% of the total number of trials were incorrect, and $1.8 \%$ were outliers.

As depicted in Fig. 1, reactions to words on the prospective list were faster than reactions to words associated with the canceled list or distractor words for both types of monitoring task. A 2 (task) $\times 3$ (word type) mixed ANOVA revealed a main effect of word type, $F(2,60)=$ $6.24, R_{\mathrm{p}}{ }^{2}=.17$. Post hoc (Bonferroni-corrected) $t$-tests confirmed an intention-superiority effect: Words on the prospective list $(M=722 \mathrm{~ms})$ were classified more quickly than words on the previously learned but canceled list $(M=$ $763 \mathrm{~ms}), t(31)=3.57, R_{\mathrm{p}}{ }^{2}=.29$. As was expected, words on the prospective list were also classified more quickly than new distractor words $(M=746 \mathrm{~ms}), t(31)=2.62, R_{\mathrm{p}}{ }^{2}=.18$. In contrast to our expectations, response latencies for words on the previously learned canceled list and new distractor words did not differ, $t<1$. Participants' overall latencies were descriptively shorter in the videoclip condition $(M=$ $697 \mathrm{~ms})$ than in the written report condition $(M=790 \mathrm{~ms})$, but the respective main effect missed the preset criterion of statistical significance, $F(1,30)=3.68, R_{\mathrm{p}}^{2}=.11, p=.07$. More important, there was no indication of a task $\times$ word type interaction, $F<1$. ${ }^{1}$

\footnotetext{
${ }^{1}$ In Experiment 1 , participants took part in groups of 2-4. Twenty participants were tested in groups of 4,6 participants in groups of 3, and another 6 participants in pairs. In order to test whether group size influenced the size of the intention-superiority effect, we computed an ANOVA with group size as the independent factor and intentionsuperiority effect (latencies for canceled list minus latencies for prospective list) as the dependent variable. There was no effect of group size, $F<1$. In Experiment 2, all participants were tested in pairs.
} 


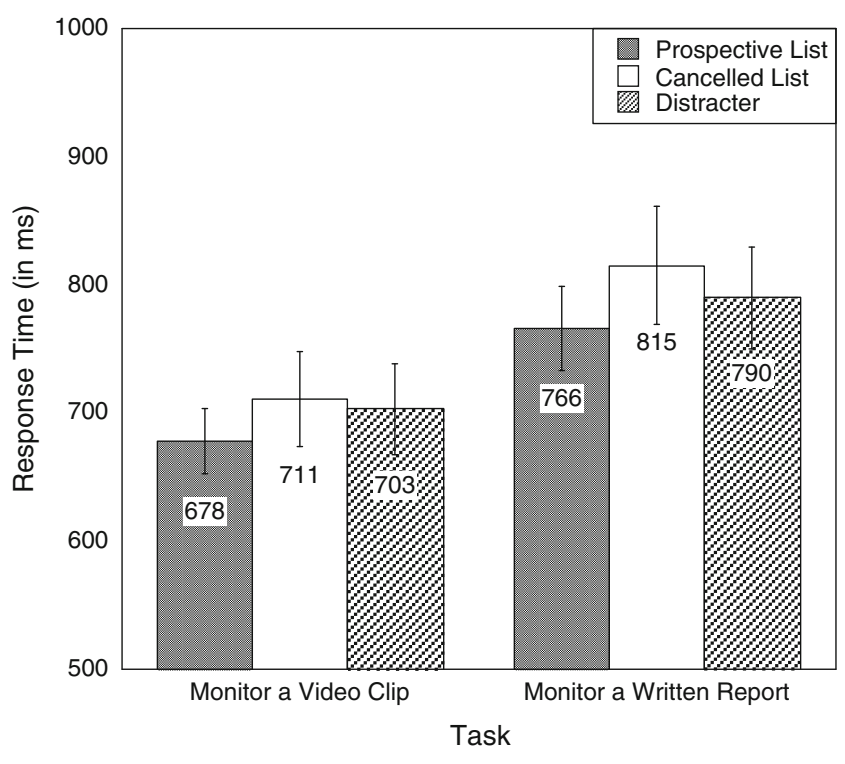

Fig. 1 Mean response latencies for words on the prospective list, words on the canceled list, and distractor words, separately for the tasks of monitoring a videoclip and monitoring a written report. All participants expected an outcome evaluation in Experiment 1. Error bars represent standard errors of the means

\section{Discussion}

Experiment 1 demonstrated an intention-superiority effect for two monitoring tasks unrelated to future enactment. For both types of monitoring tasks, words of the prospective list were responded to more quickly than words of the canceled list. We also expected that list words, having been learned only some minutes earlier, should be more easily accessible than distractor words. This was true for words on the intention-relevant list, but not for words on the canceled list. We will discuss a possible reason in the "General Discussion" section.

In previous experiments in which similar study materials were used, comparable latencies have been reported for stimuli on the prospective and neutral lists if participants were instructed to monitor someone's performance (Goschke \& Kuhl, 1993; Marsh et al., 1998) or to verbally recall these actions (Freeman \& Ellis, 2003). Thus, presenting actions as study materials is not sufficient to establish an intention-superiority effect, but participants' expectations concerning the intended task are. In contrast to other experiments that yielded no intention-superiority effect, in our experiment the quality of the task output (the grading decision) was evaluated in public. Participants expected to justify in front of others the reasons that made them give a certain grade. The quality of their grading decision was personally relevant. Under these conditions, we observed an intention-superiority effect both for the monitoring task that involved grading actions observed on a video clip and for a monitoring task maximally unrelated to enactment (i.e., monitoring a written report).

We assume that the observed intention-superiority effect in Experiment 1 reflects participants' high commitment to fulfill the intended task properly because their outcome would be evaluated. In other words, the crucial difference between our experiment, where an intention-superiority effect was observed, and previous null results appears to be outcome evaluation. What is missing to increase the strength of our argument is a direct manipulation of outcome evaluation.

\section{Experiment 2}

In Experiment 2, participants either expected to discuss their task performance with other participants or expected to fulfill the intended task with no one evaluating their task performance. Thus, the quality of task performance either was personally relevant or had no personally relevant consequences. We used two types of tasks: enactment and monitoring a recall protocol.

Previous studies have demonstrated a robust intentionsuperiority effect for an enactment task (Dockree \& Ellis, 2001; Freeman \& Ellis, 2003; Goschke \& Kuhl, 1993; Marsh et al., 1998; Marsh et al., 1999). We assume that in these experiments, the motivation to fulfill the enactment task properly was high because participants anticipated some kind of evaluation of their enactment performance by the experimenter. Consequently, we expected to replicate an intention-superiority effect for participants who were told that their enactment performance would be evaluated by other participants. Replicating Experiment 1, we also expected an intention-superiority effect for a monitoring task if an evaluation of monitoring performance was anticipated.

For participants who were told that they could realize the prospective tasks without evaluation, it was rather irrelevant how good they performed at the respective task, although they also were asked to realize the intended tasks. Similar to the observation task used by Goschke and Kuhl (1993), participants could realize the task properly, but they did not have to. Thus, if outcome evaluation is a crucial factor that increases the accessibility of intention-related materials, we should find comparable response latencies for stimuli on the prospective list and the neutral list if the task performance is not evaluated, irrespective of the type of task (enactment or monitoring). In contrast to our hypotheses, if the intention-superiority effect is mainly due to the availability of motor information, as proposed by Freeman and Ellis (2003), we should observe an intention-superiority effect for all participants who intend to enact the actions, with or without evaluation. 


\section{Method}

Participants Sixty-four participants were recruited on campus (age: $18-29$ years, $M=23, S D=3 ; 50 \%$ female). They received financial compensation for participation (3€). Sixteen participants were randomly allocated to each group.

Materials and procedure Study lists, the lexical-decision task, and to-be-monitored materials for the monitoring task were taken from Experiment 1. Two participants were tested in each session. They were asked to learn two lists of actions for future tasks. They were going to enact one studied list (enactment task) and to monitor and grade a written recall of the other list (monitoring task). Half of the participants were informed that they were going to discuss their performance with other participants. For these participants, the task outcome was evaluated as in Experiment 1. The motivation to fulfill the intended task properly should be high. The other half of the participants were informed that they could realize tasks anonymously. It was emphasized that no one would watch the enactment performance behind a partition wall and that the grading decision could not be traced back to the specific person. Although participants had to realize the intended task, the quality of their task performance (the outcome) was irrelevant.

Otherwise, the procedure was identical to that in Experiment 1. Participants studied the two lists with the intention of enacting one list and monitoring the other list. After a delay task, for half of the participants, the experimenter canceled the enactment task (thus, monitoring remained the relevant task); for the other half, the monitoring task was canceled (and the enactment task remained the relevant task). Next, participants completed the LDT. Then they indicated what task they were going to perform (enactment or monitoring), and memory for the prospective and canceled lists was tested in an unexpected free recall test. Afterwards, they realized the denoted task. Participants who expected an outcome evaluation either enacted the prospective list in the presence of their partner or gave a school grade for recall performance and, subsequently, discussed the respective task performance with their partner. Participants who expected no outcome evaluation enacted the prospective list behind a partition wall or gave a grade for the verbal report without discussing it. Finally, importance ratings of the task and demographic data were collected, and participants were debriefed.

A minor change, as compared with Experiment 1, concerned the delay task. In order to avoid social interactions between participants before task realization, we used a written task instead of a get-to-know-each-other task as the delay task. Participants were instructed to write down their opinions about different topics (e.g., studying abroad, tuition fees). The experimenter interrupted the task after 4 min, allegedly due to time constraints. This seemed a nice way to rule out the possibility that the social delay task would affect the results of Experiment 1. Participants who expected their task performance to be evaluated interacted with the other participant only after the LDT. In addition, for participants who were told that task realization was anonymous and private, a social delay task may have reduced the credibility of this manipulation.

Design Task (enactment vs. monitoring) and outcome evaluation (with vs. without) were manipulated between subjects. Word type (prospective list, canceled list, distractor) was manipulated within subjects. The main dependent variable was response latency in the lexical-decision task.

\section{Results}

Preliminary analyses Importance ratings were analyzed in a 2 (task) $\times 2$ (outcome evaluation) ANOVA. No effects were statistically significant (all $F \mathrm{~s}<3.4$; with outcome evaluation, $M=4.8$ for the enactment task and $M=4.7$ for the monitoring task; without outcome evaluation, $M=3.8$ for the enactment task and $M=5.3$ for the monitoring task, with higher values indicating higher importance ratings). The majority of participants who expected an evaluation (out of 16, 11 for the enactment task, 13 for the monitoring task), as well as those who expected no evaluation (14 for the enactment task, 11 for the monitoring task), recalled both lists perfectly. The other participants remembered at least three actions on each list.

Lexical-decision task Incorrect trials (3.8\%) and outliers $(1.2 \%)$ were removed from lexical decision data. As is shown in the upper half of Fig. 2 there was, again, an intention-superiority effect if an outcome evaluation was expected. The lower half of Fig. 2 shows that there was no indication of an intention-superiority effect if the tasks were going to be realized without further relevant consequences. In a 2 (task) $\times 2$ (outcome evaluation) $\times 3$ (word type) mixed ANOVA, the corresponding interaction of word type and outcome evaluation was significant, $F(2,120)=4.09$, $R_{\mathrm{p}}{ }^{2}=.06$. Simple main effect analyses indicated differences between word types for participants expecting an outcome evaluation, $F(2,59)=4.67, R_{\mathrm{p}}{ }^{2}=.14$, but not for participants expecting no evaluation, $F<1$. Replicating Experiment 1, when participants expected a public evaluation of their performance, words on the prospective list $(M=$ $713 \mathrm{~ms}$ ) were classified more quickly than words on the canceled list $(M=761 \mathrm{~ms}), t(31)=4.13, R_{\mathrm{p}}{ }^{2}=.35$. Again, prospective words were also classified more quickly than distractor words $(M=736 \mathrm{~ms}), t(31)=2.57, R_{\mathrm{p}}{ }^{2}=.18$, but 

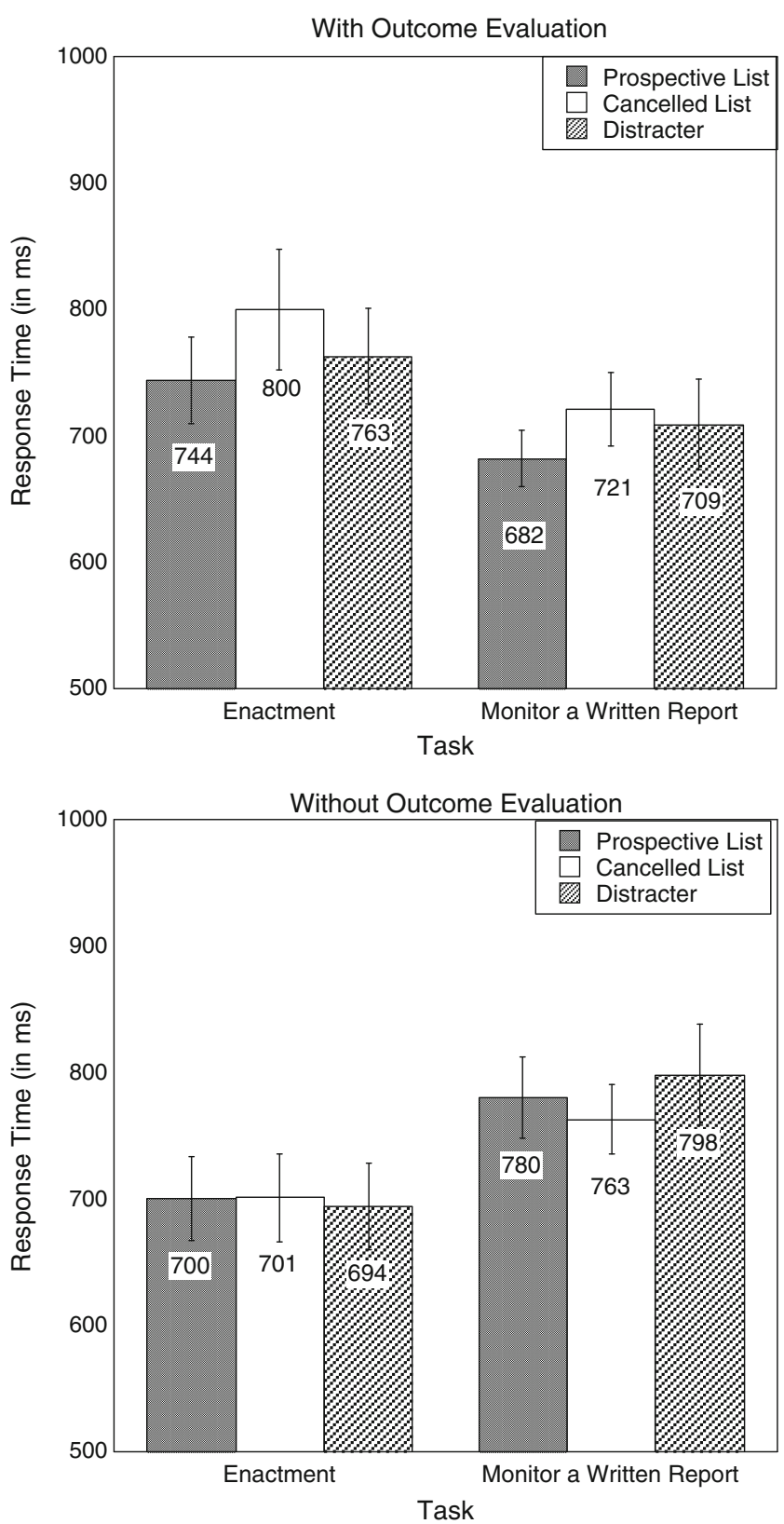

Fig. 2 Mean response latencies for words on the prospective list, words on the canceled list, and distractor words, separately for each task, enactment and monitoring a written report, and for participants expecting an outcome evaluation or no outcome evaluation in Experiment 2. Error bars represent standard errors of the means

latencies for words on the canceled list and distractor words did not differ significantly, $t(31)<1.8, p=.10$. However, if no evaluation of task performance was expected, latencies for words on the prospective list $(M=740 \mathrm{~ms})$, words on the canceled list $(M=732 \mathrm{~ms})$, and distractor words $(M=$ $745 \mathrm{~ms})$ were comparable, $t s<1.2$. There was also a task $\times$ evaluation interaction, $F(1,60)=5.46, R_{\mathrm{p}}^{2}=.08$, indicating differences between groups in overall reaction time. More important, neither the task $\times$ word type interaction nor the three-way interaction was significant, $F_{\mathrm{S}}<1$.

\section{Discussion}

The results of Experiment 2 are in line with the assumption that motivational aspects - here, the expectation of an outcome evaluation - contribute to the intention-superiority effect. Replicating Experiment 1, we observed an intentionsuperiority effect if task realization was going to be evaluated by others. In contrast, when task realization had no consequences, there was no indication for faster reactions to intention-relevant stimuli. The null finding for the enactment task without evaluation is particularly interesting. If the availability of motor information determined the intention-superiority effect, there should have been a reaction-time advantage for to-be-enacted materials, relative to neutral materials, irrespective of the evaluation situation.

The ratings on the importance of the intended task did not differ significantly between groups. Yet, descriptively, the enactment task without evaluation was rated less important than the enactment task with relevant consequences in terms of an evaluation. At first sight, this finding supports the assumption of an intention-superiority effect for more important tasks. However, if task importance per se was central, we should also have observed an intentionsuperiority effect in both monitoringtask conditions. The importance ratings for the monitoring task without evaluation were descriptively even higher than the ratings for the same task with relevant consequences. It thus seems that reported task importance is not a reliable proxy for the consequences of task realization in terms of an evaluation; the latter were essential for a heightened accessibility.

\section{General discussion}

The main hypothesis brought forward in the present research was that motivational aspects, such as expectation of an outcome evaluation or relevance of an intended task, contribute to the intention-superiority effect. In two experiments, we demonstrated a heightened accessibility for intention-relevant stimuli, relative to other learned materials, when task realization had personally relevant consequences in terms of an evaluation of the task performance. This intention-superiority effect was independent of the type of prospective task. There was an intention-superiority effect if task realization was checked in terms of an outcome evaluation, and thus, presumably, participants' commitment to fulfill the task properly was high. Our findings are consistent with theories that consider intentions as motivational states characterized by enhanced accessibility (Förster et al., 2005; e.g., Lewin, 1926). Although a strategic allocation of attentional resources also may have strengthened the accessibility of intention-relevant material during 
postponement, we think the main source of activation is motivational. As Experiment 2 clearly demonstrates, heightened activation was restricted to tasks associated with personally relevant consequences (here, an outcome evaluation), be they related to the execution of actions or not.

In contrast to other studies (Marsh et al., 1998; Marsh et al., 1999; but see Dockree \& Ellis, 2001, Experiment 1), in our experiments responses to previously studied words were not generally faster than responses to new distractor words in the lexical-decision task. Although words of the previously learned prospective list were classified more quickly than new distractor words, response latencies for words of the previously learned but canceled list did not differ significantly from new distractor words. Considering Marsh et al.'s (1999) findings of longer latencies for canceled materials, relative to other learned materials, an inhibitory account may explain our finding. Initial activation and subsequent inhibition may have led to response latencies comparable to those for new distractor words. Inhibiting information associated with the canceled task may be adaptive in order to avoid an accidental initiation of task execution when intentions become impossible to complete (e.g., going to stop at a library to borrow a book beyond opening hours) (for a more detailed discussion of inhibitory processes, see Förster et al., 2007).

In everyday life, the announced evaluation of one's performance in an intended task is a rather uncommon means for ensuring prospective remembering. We used an evaluation announcement for several reasons. First, it allowed us to manipulate the type of intended task (enactment or monitoring) while holding the consequences of failure (a poor performance feedback) constant. Second, the announced evaluation ensured that the experimenterdefined tasks were personally relevant for participants. Third, we think the typical postponed enactment task can be described as an evaluation situation. It therefore seemed reasonable to design other tasks that shared features with a laboratory task that reliably shows an intention-superiority effect. Future research should use more naturalistic manipulations to increase or decrease task relevance. In both of the present experiments, participants' rating of task importance was not associated with the size of the intentionsuperiority effect. Thus, stressing the importance of the intended task itself might not be sufficient (or subjective ratings are not reliable proxies of importance as indicated by activation). As Experiment 2 suggests, a manipulation of task importance in terms of anticipated negative consequences of failure is more promising. Another possibility for strengthening the commitment to fulfill a task is providing benefits for successful task realization. For instance, Förster et al. (2005) reported shorter response latencies for intention-related stimuli when intention reali- zation was associated with a high monetary incentive $(1 €)$, but not when associated with a low incentive $(0.05 €)$.

In contrast to previous work where participants were tested in the presence of an experimenter (e.g., Freeman \& Ellis, 2003; Goschke \& Kuhl, 1993; Marsh et al., 1998; Marsh et al., 1999), in our experiments, task realization involved the interaction with other participants. We assume that the presence of another participant does not affect participants differently than the presence of an unknown experimenter. The mere presence of others is not sufficient to influence someone's performance, but motivational factors are, such as evaluation apprehension (e.g., Cottrell, 1968). We demonstrated an intention-superiority effect when participants' anticipation of a (socially) relevant evaluation of their performance was high. Although the observation task in previous work (Goschke \& Kuhl, 1993; Marsh et al., 1998) was also a social situation, an evaluation of task performance was unlikely, since no appraisable outcome was produced. Low evaluation apprehension in these observation tasks may explain the finding of comparable response latencies for intention-relevant and neutral stimuli. We think earlier work on the intentionsuperiority effect can easily be interpreted in terms of a heightened activation of motivation-related representations. In those studies, either task realization was linked to an (implicit) evaluation of one's own enactment performance (Goschke \& Kuhl, 1993; Marsh et al., 1998; Marsh et al., 1999), or remembering to do something was important for others (Dockree \& Ellis, 2001). It may be that materials associated with prospective tasks that occur in a social context or carry implication for social relationships are particularly easily accessible (cf. Winograd, 1988). It would be interesting to test whether an intention-superiority effect can be obtained with other tasks that do not involve social interactions but are personally relevant.

Freeman and Ellis (2003) provided some evidence that the intention-superiority effect is based on the activation and storage of motor information when an intention for future execution is formed (see also Eschen et al., 2007, for supporting data in an fMRI study). The general idea is that actions verbally encoded for future enactment elicit similar representations as actions carried out during encoding. In both cases, motor-relevant information is activated that facilitates recall. Following this reasoning, an enactment intention would be crucial for an intention-superiority effect. The data pattern we observed is not compatible with that account, because anticipated outcome evaluation, not enactment intention, determined the intention-superiority effect that we found (see Experiment 2). We cannot exclude the possibility that the materials we chose stimulated the encoding of motor information at study, since study lists described simple distinct activities. However, speaking against this idea, other experiments that used lists of action 
phrases for an observation task (Goschke \& Kuhl, 1993; Marsh et al., 1998) or lists of verbs for verbal recall (Freeman \& Ellis, 2003) showed no reaction-time difference. In sum, the missing intention-superiority effect for an enactment task without outcome evaluation that we found, and the intention-superiority effect for monitoring tasks unrelated to enactment, challenge the proposition that motor processes are always involved in the activation of intentions in a postponed-intention paradigm.

We think the increased activation in the postponedintention paradigm reflects a more general phenomenon: heightened accessibility of intention-relevant information during postponement. For example, Förster et al. (2005) instructed participants to get up and notify the experimenter when they detected a certain object combination in a series of pictures. Between blocks of pictures, participants performed anLDT. Instead of using stimuli describing the content of the intention - that is, the intended actions (e.g., to get up) - they used stimuli associated with the cue that indicates an appropriate context to realize the intention. Similar to an intention-superiority effect for stimuli of the intention-relevant actions, the authors demonstrated shorter latencies to words associated with the intention-relevant cue. Interestingly, in this research, the prospective task (notifying the experimenter) could also be perceived as a personally relevant situation. Again, there was an appraisable outcome (whether participants detected the target combination or not) and an evaluator (the experimenter). In a similar vein, Fitzsimons and Shah (2008) demonstrated that names of other people who are instrumental for achieving a specific goal are more easily accessible when the goal is activated. Again, information that may help to realize an intention successfully was more easily accessible.

To conclude, whereas future overt enactment at first appeared to be the relevant factor affecting the accessibility of intention-related stimuli, the present experiments show that factors presumably affecting motivation, such as anticipated outcome evaluation, determine the activation of intention-related materials. Whenever task performance matters, there is an intention-superiority effect. Thus, it appears that a more general mechanism than has previously been suggested is at work: Our cognitive system is tuned to materials associated with any important prospective task, be it an enactment task or not.

\section{References}

Cohen, J. (1977). Statistical power analysis for the behavioral sciences (Rev. ed.). Hillsdale, NJ: Erlbaum.

Cottrell, N. B. (1968). Performance in the presence of other human beings: Mere presence, audience, and affiliation effects. In E. C. Simmel, R. A. Hoppe, \& G. A. Milton (Eds.), Social facilitation and imitative behaviour (pp. 91-110). Boston: Allyn \& Bacon.
Dockree, P. M., \& Ellis, J. A. (2001). Forming and canceling everyday intentions: Implications for prospective remembering. Memory \& Cognition, 29, 1139-1145.

Ellis, J. (1996). Prospective memory or the realization of delayed intentions: A conceptual framework for research. In M. Brandimonte, G. O. Einstein, \& M. A. McDaniel (Eds.), Prospective memory: Theory and application (pp. 1-22). Mahwah, NJ: Erlbaum.

Engelkamp, J. (1997). Memory for to-be-performed tasks versus memory for performed tasks. Memory \& Cognition, 25, 117-124.

Eschen, A., Freeman, J., Dietrich, T., Martin, M., Ellis, J., Martin, E., et al. (2007). Motor brain regions are involved in the encoding of delayed intentions: A fMRI study. International Journal of Psychophysiology, 64, 259-268.

Fitzsimons, G. M., \& Shah, J. Y. (2008). How goal instrumentality shapes relationship evaluations. Journal of Personality and Social Psychology, 95, 319-337.

Förster, J., Liberman, N., \& Friedman, R. S. (2007). Seven principles of goal activation: A systematic approach to distinguishing goal priming from priming of non-goal constructs. Personality and Social Psychology Review, 11, 211-233.

Förster, J., Liberman, N., \& Higgins, E. T. (2005). Accessibility from active and fulfilled goals. Journal of Experimental Social Psychology, 41, 220-239.

Freeman, J. E., \& Ellis, J. A. (2003). The representation of delayed intentions: A prospective subject-performed task? Journal of Experimental Psychology: Learning, Memory, and Cognition, 29, 976-992.

Goschke, T., \& Kuhl, J. (1993). Representation of intentions: Persisting activation in memory. Journal of Experimental Psychology: Learning, Memory, and Cognition, 19, 1211-1226.

Koriat, A., Ben-Zur, H., \& Nussbaum, A. (1990). Encoding information for future action: Memory for to-be-performed tasks versus memory for to-be-recalled tasks. Memory \& Cognition, $18,568-578$.

Lewin, K. (1926). Vorsatz, Wille und Bedürfnis. Psychological Research, 7, 330-385.

Marsh, R. L., Hicks, J. L., \& Bink, M. L. (1998). Activation of completed, uncompleted, and partially completed intentions. Journal of Experimental Psychology: Learning, Memory, and Cognition, 24, 336-349.

Marsh, R. L., Hicks, J. L., \& Bryan, E. S. (1999). The activation of unrelated and canceled intentions. Memory \& Cognition, 27, 320-327.

Meacham, J. A. (1988). Interpersonal relations and prospective remembering. In M. M. Gruneberg, P. E. Morris, \& R. N. Sykes (Eds.), Practical aspects of memory: Current research and issues. Vol. 1: Memory in everyday life (pp. 354-359). Oxford: Wiley.

Saltz, E. (1988). The role of motoric enactment (M-processing) in memory for words and sentences. In M. M. Gruneberg, P. E. Morris, \& R. N. Sykes (Eds.), Practical aspects of memory: Current research and issues. Vol. 1: Memory in everyday life (pp. 408-414). Oxford: Wiley.

Winograd, E. (1988). Some observations on prospective remembering. In M. M. Gruneberg, P. E. Morris, \& R. N. Sykes (Eds.), Practical aspects of memory: Current research and issues. Vol. 1: Memory in everyday life (pp. 349-353). Oxford: Wiley.

The writing of this article was supported by grants from the Deutsche Forschungsgemeinschaft (German Science Foundation, Ste 938/7-1, 7-2). We thank Christine Johannes for valuable comments and Dave Cromm, Linda Köhler, and Sascha Poppitz for support with data collection. 